\title{
Comparison of Particle Sizing Techniques in the Case of Inhalation Dry Powders
}

\author{
CYNTHIA BOSQUILLON, CATHERINE LOMBRY, VERONIQUE PREAT, RITA VANBEVER \\ Université catholique de Louvain, School of Pharmacy, Department of Pharmaceutical Technology, Avenue E. Mounier, \\ 73 UCL 73.20, 1200 Brussels, Belgium
}

Received 25 October 2000; revised 9 March 2001; accepted 15 March 2001

\begin{abstract}
The objectives of this work were (i) to validate electrical zone sensing and laser diffraction for the analysis of primary particle size in the case of inhalation dry powders and (ii) to study the influence of the aggregation state of the powder on the sizing techniques. Free-flowing dry powders were prepared by spray-drying with a combination of albumin, lactose, and dipalmitoylphosphatidylcholine. The replacement of lactose by mannitol, the removal of albumin, and the atomization at high relative humidity all increased powder cohesion. Automated measurements were compared with primary particle sizes collected by light and electron microscopy. The mass mode obtained by electrical zone sensing and the mass median diameter measured by laser diffraction following dispersion with compressed air at a pressure of 3 bar or following suspension in water and ultrasonic dispersion at a power of $60 \mathrm{~W}$ for $30 \mathrm{~s}$ each provided primary particle sizes close to microscopy measurements. However, these conditions only applied in the case of slightly to moderately aggregated powders. For strongly agglomerated powders, an exact measurement of the size was only collected by laser diffraction in the wet state combined with ultrasonic dispersion. Our study underlies how measurement of primary particle size highly depends on both powder material and proper particle dispersion. ( 2001 Wiley-Liss, Inc. and the American Pharmaceutical Association J Pharm Sci 90:2032-2041, 2001
\end{abstract}

Keywords: primary particle size; inhalation dry powder; laser diffraction; electrical zone sensing; microscopy

\section{INTRODUCTION}

A particle entrained in the respiratory tract will deposit in a well-defined region of the lungs that will depend on the aerodynamic diameter of the particle as well as on ventilatory conditions. ${ }^{1}$ The aerodynamic diameter of a spherical particle, $d_{\text {aer }}$, is related to its geometric diameter, $d$, as well as density, $\rho$, according to the formula $d_{\text {aer }}=d \sqrt{ } \rho / \rho_{1}$, where $\rho_{1}=1 \mathrm{~g} / \mathrm{cm}^{3}{ }^{2}$ Particles with a $d_{\text {aer }}>10 \mu \mathrm{m}$ do not go beyond the oropharyngeal region. Particles with a $d_{\text {aer }}$ between 5 and $10 \mu \mathrm{m}$ mainly

Correspondence to: R. Vanbever (Telephone: 32276473 25; Fax:. 32276473 98; E-mail: vanbever@farg.ucl.ac.be)

Journal of Pharmaceutical Sciences, Vol. 90, 2032-2041 (2001)

(C) 2001 Wiley-Liss, Inc. and the American Pharmaceutical Association deposit by inertial impaction in the trachea and upper airways where velocities are maximum. ${ }^{3,4}$ The effect of gravitational settling is most pronounced in the central and distal tract and occurs in case of $1-5-\mu \mathrm{m} d_{\text {aer }}$ particles. Finally, submicrometer-size particles are exhaled and/or deposit by random Brownian motion in distal regions. Selective deposition of drugs within the respiratory tract can allow therapeutic targeting of a disease that has predilection for a specific location (e.g., the tracheo-bronchial tree in the case of cystic fibrosis) or promote systemic absorption by deposition in the alveolar region.

The aerodynamic diameter of aerosol particles can be calculated by separately measuring $d$ and $\rho$ but can also be assessed experimentally using benchmark cascade impactor methods. ${ }^{5,6}$ 
Although the computed primary $d_{\text {aer }}$ allows a first evaluation of particle size adequacy for pulmonary delivery, the experimental mass median aerodynamic diameter (MMAD) takes account of particle aggregates and gives therefore the ultimate aerodynamic behavior of the aerosol. In addition, comparison of MMAD with $d_{\text {aer }}$ brings information on dry powder cohesiveness and aerosolization properties.

The notion of aerodynamic diameter is central in particle deposition in the lungs and therefore in pulmonary drug delivery. The specific aim of this article was to obtain an exact estimation of the primary particle geometric diameter, $d$, involved in this physical property in the particular case of inhalation dry powders. We compared electrical zone sensing and laser diffraction following dispersion in the dry and wet state to light and electron microscopy for the analysis of dry powders particle sizes. Electrical zone sensing is based on an electrical phenomenon. The samples are suspended in a conducting liquid which is pumped through a small orifice, on either side of which are electrodes. As a particle passes through the orifice, the resistance between the electrodes increases proportionally to the particle volume, producing a voltage pulse that is amplified, recorded, and analyzed. In laser diffraction, the dispersed particles are exposed to a laser beam that is diffracted with an angle depending on the particle size; that is, the smaller the particle, the higher the angle. ${ }^{8,9}$

Here, we determined the adequate measurement conditions of those techniques for inhalation dry powders presenting different degrees of aggregation, considering microscopy as a reference. The powders were prepared by spray-drying using GRAS (generally recognized as safe) excipients; that is, excipients that are endogenous to the lungs, such as dipalmitoylphosphatidylcholine (DPPC) and albumin, FDA-approved for pulmonary delivery as lactose, and/or the small polyol, mannitol. ${ }^{10,11}$ The behavior of the powders in the Andersen cascade impactor and the observation of their size distribution profiles in electrical zone sensing were used to evaluate the aggregation state of the powder.

\section{MATERIALS AND METHODS}

\section{Chemicals}

Human serum albumin (fraction V, 96-99\% albumin), D-mannitol, and 96\% ethanol were obtained from Sigma, and $\alpha$-lactose monohydrate was from Acros Organics (Fair Lawn, NJ). DPPC was purchased from Lipoid (Lipoid GMBH, Ludwigshafen, Germany) and coumarin from ICN (ICN Biomedicals Inc., Aurora, OH).

\section{Formulation of the Dry Powders}

Dry powders were made with DPPC, albumin, lactose, and/or mannitol, by spray-drying as previously described. ${ }^{11}$ Briefly, albumin, lactose, and/or mannitol were dissolved in distilled water. The $\mathrm{pH}$ was then adjusted to 7 by addition of $\mathrm{NaOH}$ (Merck Eurolab, Leuven, Belgium). DPPC and coumarin were dissolved in $96 \%$ ethanol. Coumarin was incorporated in the powder at a low load $(0.1 \% \mathrm{w} / \mathrm{w})$ to allow the quantification of the amount of powder deposited on each stage of the Andersen cascade impactor (see below). The water and ethanol solutions were then combined to form an 80 or $90 \%$ ethanolic solution of $0.1 \% \mathrm{w} / \mathrm{v}$ total powder concentration, ready for spray-drying processing. Solutions were pumped into the drying chamber of a laboratory-scale spray-dryer (Lab-Plant Limited, Huddersfield, England) at a rate of $20 \mathrm{~mL} / \mathrm{min}$ and pneumatically atomized through a two-fluid external mixing $0.5-\mathrm{mm}$ nozzle using compressed air at 0.1 or 1.5 bar. The inlet temperature was established at 100 or $110^{\circ} \mathrm{C}$. The outlet temperature depended on the inlet temperature and the liquid and gas flow rates, and varied between 45 and $55^{\circ} \mathrm{C}$. Spray-drying was carried out at a room relative humidity (RH) of $<40 \%$, except in the case of the preparation of mannitol/DPPC powders for which the $\mathrm{RH}$ was $50 \%$. The powders were collected and stored in a dessicator (i.e., at room temperature and $25 \% \mathrm{RH}$ ) until analysis.

\section{Primary Aerodynamic Diameter}

The theoretical aerodynamic diameter of the individual particles, $d_{\text {aer }}$, was calculated based on the following definition: ${ }^{2}$

$$
d_{\mathrm{aer}}=\sqrt{\frac{\rho}{\rho_{1}}} d
$$

where $\rho=$ particle density, $\rho_{1}=1 \mathrm{~g} / \mathrm{cm}^{3}$ and $d=$ mass median particle diameter as measured by light microscopy, which was the reference technique applicable to each powder considered (see below).

The particle density, $\rho$, was evaluated by tap density measurements; that is, following 
1000 taps which allowed the density to plateau. ${ }^{7,8}$ Assuming an efficient packing, the tap density of a monodisperse assay of spheres is a 21\% underestimate of the true particle density because of the void spaces between particles. Although, polydispersity of powders may reduce the void volume between particles, it is probably counterbalanced by less than perfect packing. ${ }^{10}$

\section{In Vitro Aerosol Deposition in the Andersen Cascade Impactor}

The pulmonary deposition of the dry powders was investigated in vitro using an Andersen cascade impactor (1 ACFM Eight Stage Non-Viable Cascade Impactor, Graseby Andersen, Atlanta, GA) at $30-40 \%$ room $\mathrm{RH}$, as previously described. ${ }^{11}$ Briefly, the trays of the impactor were coated with a hydroxypropylmethylcellulose gel $(22.5 \% \mathrm{w} / \mathrm{v}$ in water). A hard gelatin capsule (size 2, Capsugel) previously stored in a dessicator for at least 2 days, was filled with the powder to $\sim 50 \%$ of its volume and placed in a Spinhaler ${ }^{\mathrm{TM}}$ inhaler (Fisons, Bedford, MA). The capsule was then pierced, and the liberated powder was drawn through the impactor operating at a flow rate of $28.3 \mathrm{~L} / \mathrm{min}$ (the flow rate at which the Andersen impactor is calibrated) for $10 \mathrm{s.}{ }^{5}$ The amount of powder deposited on the different levels was recovered by immersing each tray and the stage below in $80 \%$ ethanol. The powder deposited in the throat and on the pre-separator was also collected. After dissolution of the particles, the fluorescence due to coumarin of each solution was determined with a Perkin Elmer Luminescence Spectrometer LS50B $\left(\lambda_{\mathrm{ex}}=458 \mathrm{~nm}\right.$, $\lambda_{\text {em }}=545 \mathrm{~nm}$ ).

The emitted dose (ED) was determined as the percent of total powder mass exiting the capsule. The cumulative mass of powder less than the stated size of each stage of the Andersen impactor was calculated and plotted on a log probability scale as percent of total mass recovered in the impactor against the effective cutoff diameter. According to the European pharmacopoeia, ${ }^{5}$ the experimental MMAD of the particles is defined from this graph as the particle size at which the line crosses the 50\% mark and the geometric standard deviation (GSD), as GSD

$$
=\sqrt{\frac{\text { size } \mathrm{X}}{\text { size } \mathrm{Y}}}
$$

where size $\mathrm{X}$ is the particle size for which the line crosses the $84 \%$ mark and size $\mathrm{Y}$ is the $16 \%$ mark.
The fine particle fraction (FPF) was calculated by interpolation from the same plot as the fraction of powder emitted from the inhaler with an aerodynamic size $\leq 5 \mu \mathrm{m} .^{5,11}$ The GSD values of the powders prepared (see Table 1) ranged between 1.4 and 1.8 .

\section{Electron and Light Microscopy}

The powders were visualized using a conventional scanning electron microscope (SEM; Philips CM12/STEM, Eindhoven, The Netherlands). The dry powders were mounted onto metal plates and a 10-nm-thick gold film was sputter coated on the samples with a Balzers SCA 020 (Balzers Union, Liechtenstein) before visualization. The projected area of 100 particles was collected by surrounding them manually, and the analysis was performed with image analysis software (AnalySIS, SoftImaging Software Gmbh).

The powders were also viewed using a light microscope (Ortholux, Leitz Wetzlar, Germany). The samples were mounted on slides without further preparation before observation. The diameter of 100 particles was estimated visually. Most of the particles appeared spherical under the light microscope, except for particles of powder A for which the bigger diameter was taken into account. The mass median particle diameter was calculated from the 100 particle diameters collected with both the electron and light microscopy techniques.

\section{Electrical Zone Sensing}

The powders were suspended in saline (Isotone II, Analis, Namur, Belgium) in a microfuge tube (Eppendorf, Netheler-Hinz-Gmbh, Germany), and the suspension was mixed by vortex for $1 \mathrm{~min}$ before measurement. A few droplets of the suspension were added to the counting cell of the Coulter Multisizer II (Coulter Electronics, Luton, England), and the number distribution was as a function of particle size collected using an orifice tube with a diameter of $70 \mu \mathrm{m} .^{8,9}$ The mass size distribution was then indirectly obtained by calculation. The size distribution was analyzed by an AccuComp Color Software Version 1.10 (1990; Coulter Electronics Inc, Hialeah, FL) and both the mass median and the mass mode were noted.

\section{Laser Diffraction}

Measurements were made both in the dry state and in suspension in water. In the dry state mode, 


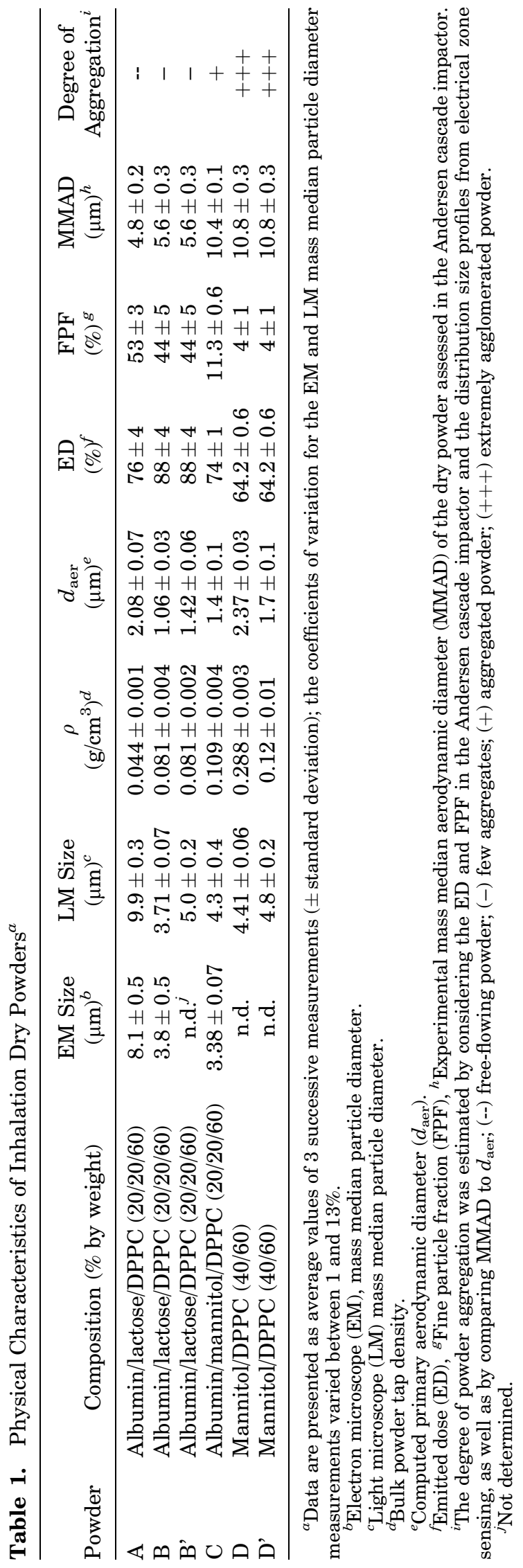

the powders were dispersed with compressed air at varying pressure $(0.25-6$ bar) through a venturi tube (RODOS, Sympatec GmbH, ClausthalZellerfelg, Germany) before sizing with a laser diffractometer (HELOS, Sympatec). In the wet state mode, the samples were suspended in water in a $50-\mathrm{mL}$ glass cuvette and stirred with a magnetic bar. The particle size was measured (HELOS, Sympatec) immediately after suspension or following increasing periods of sonication $(30 \mathrm{~s}$ to $8.5 \mathrm{~min})$ at a power of $60 \mathrm{~W}$ with an $8.5-\mathrm{mm}$ diameter ultrasound tip (CUVETTE, Sympatec). For both methods, an R2 lens (Sympatec), allowing measurements in the range 0.25 $87.5 \mu \mathrm{m}$, was used. The particle size analysis was performed with WINDOX 3.4 software, and the mass median particle diameter was considered.

\section{Data Analysis}

The mass size distributions of the particles were not perfectly log-normal (Kolmogorov-Smirnov, $p>0.05$ ). Therefore, the mass median was considered instead of the mass geometric mean. Moreover, the mass median is less affected by extreme values in case of skewed distributions.

The mass median and mass mode diameters are presented as average values of three determinations [ \pm standard deviation (SD)] and were compared by Anova and $\mathrm{t}$ test (Table 1, Figures 3-6). The SDs are not shown in each figure for clarity.

\section{RESULTS}

\section{Characteristics of the Dry Powders Investigated}

Four types of dry powders with different degrees of aggregation were prepared. Difference in cohesiveness was achieved by changing powder composition and spray-drying conditions. ${ }^{11}$ The state of aggregation of the powders was estimated by the behavior in the Andersen cascade impactor, by the comparison of the experimental MMAD to the computed $d_{\text {aer }}$, as well as by the observation of the mass size distribution profiles in the electrical zone sensing technique.

Some physical characteristics of the powders are presented in Table 1. All the powders presented a low bulk powder tap density $(\rho<0.3 \mathrm{~g} /$ $\mathrm{cm}^{3}$ ), microscopy sizes between 3 and $10 \mu \mathrm{m}$, and $d_{\text {aer }}$ between 1 and $3 \mu \mathrm{m}$; and were therefore theoretically suited for administration to the deep lungs. Combining lactose, albumin, and DPPC 
(albumin/lactose/DPPC 20/20/60 w/w/w) led to dry powders with excellent aerosolization properties in the Andersen cascade impactor (powders A and $B$ ) and which exhibited narrow mass distribution size profiles in electrical zone sensing (Figure 1a). ${ }^{10,11}$ Powder A exhibited closer MMAD and $d_{\text {aer }}$ values than powder $\mathrm{B}$; the particular dispersibility of powder A likely originated from its large geometric size. ${ }^{12}$ Replacing lactose by mannitol strongly decreased flowability characteristics (powder $\mathrm{C}$ ). The additional removal of albumin and the atomization at high relative humidity led to highly aggregated powders (powder D, made of mannitol/DPPC), as demonstrated by the Andersen cascade impactor data and the distorted mass distribution size profile obtained by electrical zone sensing (Table 1, Figure 1b).

\section{Electron and Light Microscopy}

The mass median particle diameter of the four powder types was measured both by electron and light microscopy. Those techniques were considered as references because they allow individual particles to be distinguished from aggregates (Figure 2). However, it was impossible to evaluate the size of powder $\mathrm{D}$ by electron microscopy because the number of individual particles as compared with the aggregates was too small in the limited amount of powder deposited on the

a

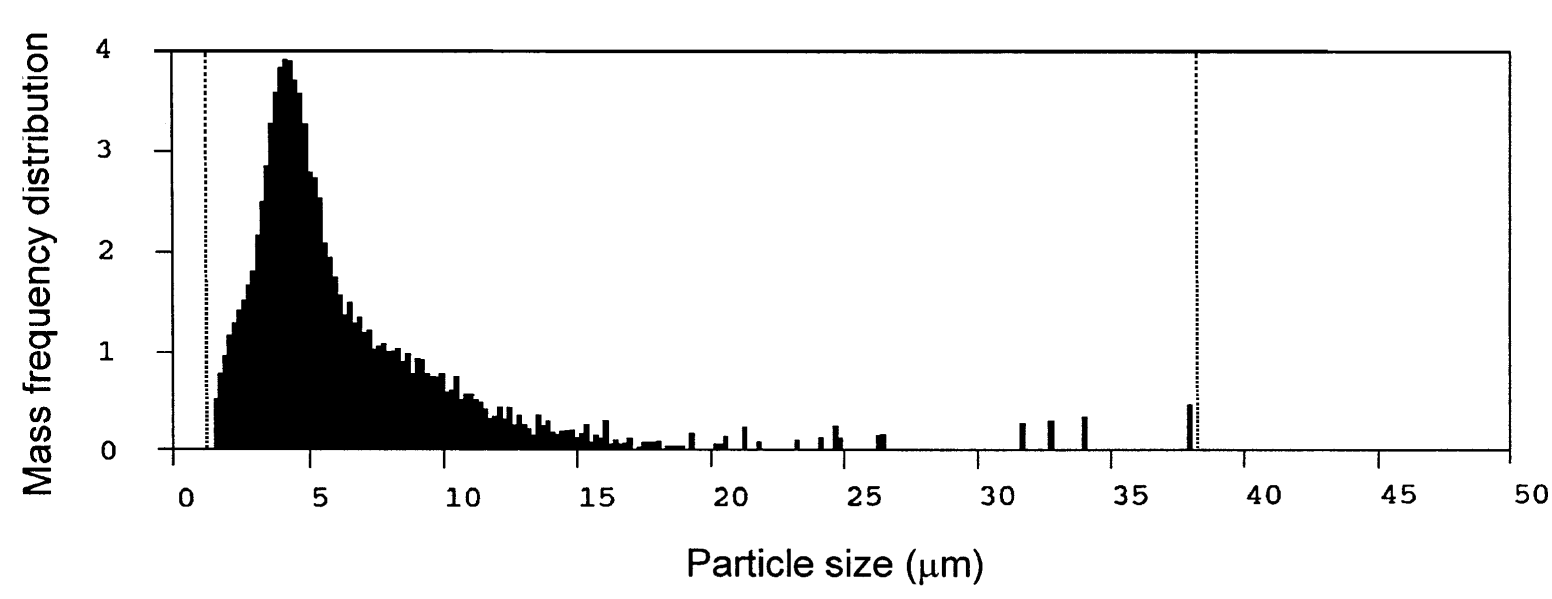

b

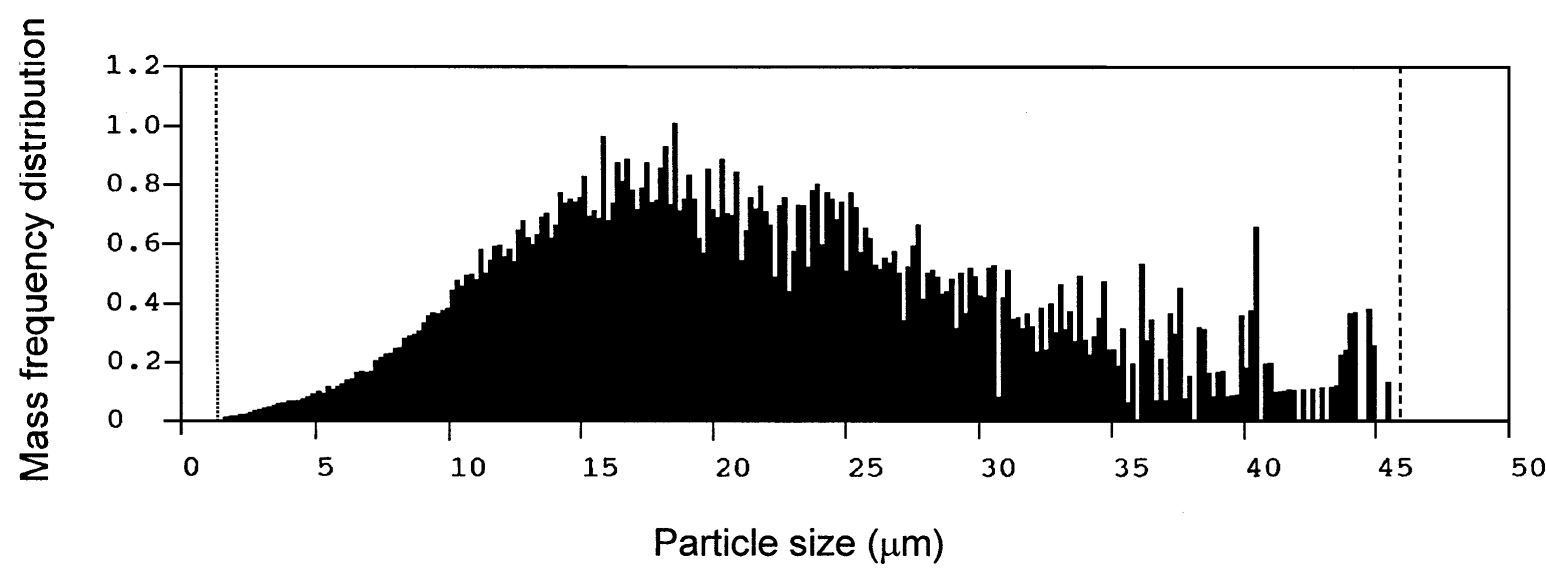

Figure 1. Mass distribution size profiles of powders B (a) and D (b) collected by electrical zone sensing. Powder B, made of albumin/lactose/dipalmitoylphoshpatidylcholine $20 / 20 / 60 \mathrm{w} / \mathrm{w} / \mathrm{w}$, was highly respirable and exhibited a narrow unimodal size profile. In contrast, powder D, made of mannitol/dipalmitoylphosphatidylcholine $40 / 60 \mathrm{w} / \mathrm{w}$, was very cohesive and the aggregates totally distorted the mass distribution ofsize. 


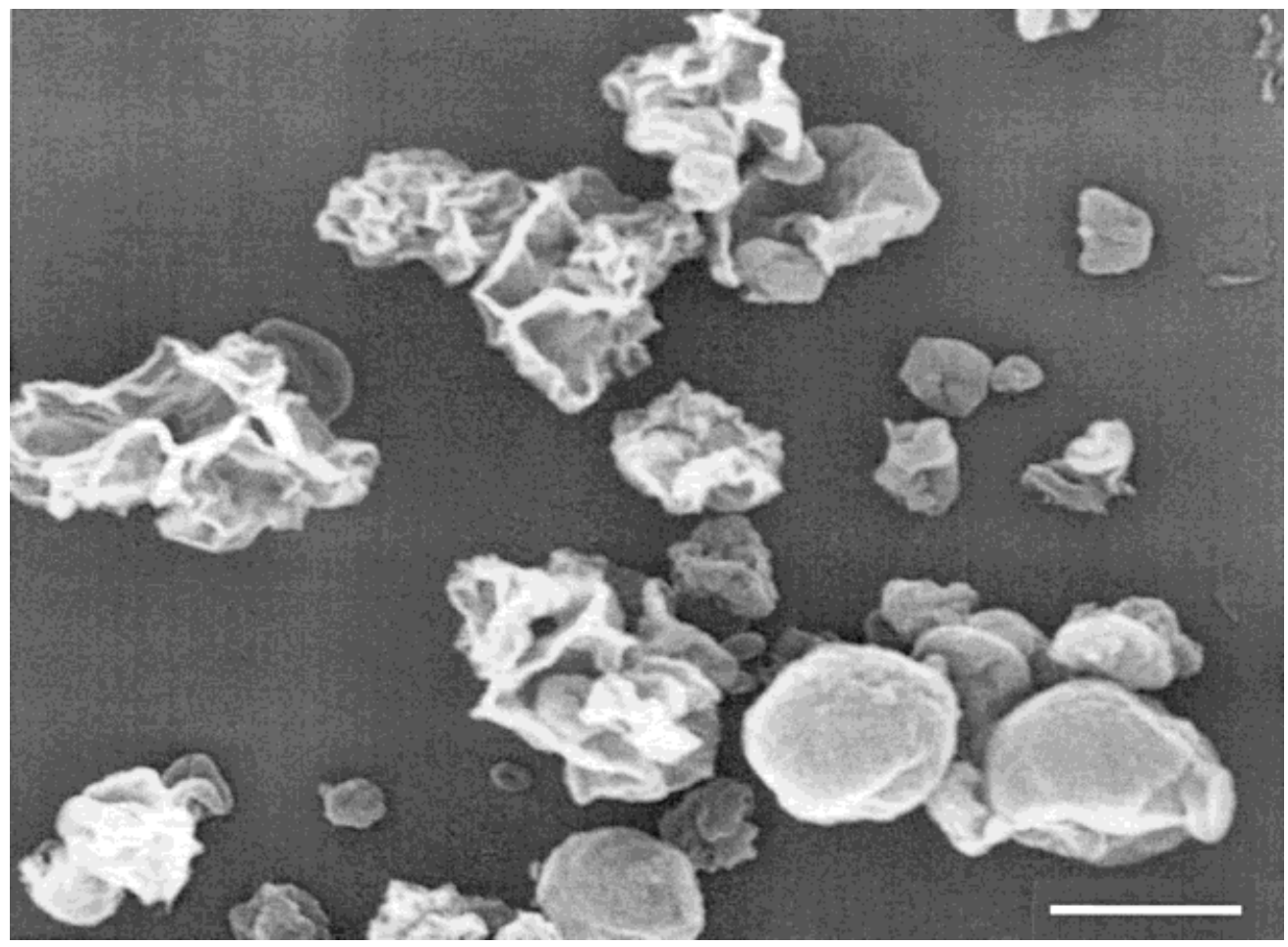

Figure 2. Scanning electron microscopy image of powder B, made of albumin/lactose/ dipalmitoylphoshpatidylcholine $20 / 20 / 60 \mathrm{w} / \mathrm{w} / \mathrm{w}$. Scale bar is $5 \mu \mathrm{m}$. The particles presented a combination of sponge-like and spherical shapes!.

metal plates. By contrast, enough isolated particles of powder D could be found by light microscopy because of the much larger amount of powder laid on the slide. The diameter obtained by light microscopy was equal to or larger than that measured by electron microscopy (Table 1; $p<0.05$ for powders A and C, $p>0.05$ for powder B). Some overestimation of particle size by light microscopy might come from the detection limit of $1 \mu \mathrm{m}$ of this technique as well as, in the case of powder A, from the measurement of the bigger diameter of the not totally round-shaped particles.

\section{Comparison of Electrical Zone Sensing and Microscopy}

The mass median and the mass mode obtained by electrical zone sensing were equal to the electron microscopy sizes only in the case of powders exhibiting a low degree of aggregation (Figure 3; powders $\mathrm{A}$ and $\mathrm{B}, p>0.05$ ). The mass median diameter of moderately cohesive powders (powder C) appeared much larger than the microscopic mass median diameter $(p<0.05)$, in contrast to the mass mode that was equal to the light microscopy mass median diameter $(p>0.05)$. The mass mode of electrical zone sensing was therefore considered the best estimation of the mass diameter of slightly to moderately aggregated particles. However, in the case of the less respirable powder (powder $\mathrm{D}$ ), the mass size distribution was greatly distorted by the agglomerates and the mode was also completely aberrant (Figures $1 b$ and 3).

\section{Comparison of Laser Diffraction and Microscopy}

For all the powders investigated, the mass median diameter measured by laser diffraction in the dry state rapidly decreased with increasing the pressure of compressed air used to deaggregate the powder and reached a slower downward trend starting at 3 bar (Figure 4). The sizes obtained following dispersion with air at a pressure $<3$ bar were superior to the microscopy sizes $(p<0.05)$, suggesting that pressures $<3$ bar were ineffective at separating the aggregates. A pressure of 3 bar allowed the diameter to get similar to microscopy measurements in the case of slightly to moderately aggregated powders $(p>0.05$ for powders A, B, B', and C); beyond 3 bar particle 


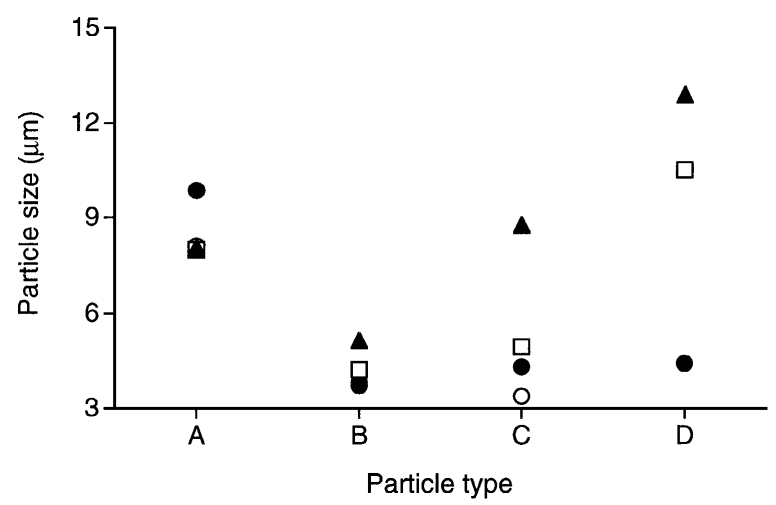

Figure 3. Comparison between electrical zone sensing (EZS) and microscopy for the analysis of particle size of powders A, B, C, and D. Key: ( $\mathbf{\Lambda}$ ) EZS mass median particle diameter; ( $\square$ ) EZS mass mode particle diameter; ( ) light microscopy mass median particle diameter; $(\bigcirc)$ electron microscopy mass median particle diameter. The coefficients of variation for the EZS mass mode measurements ranged between 1.8 and $7 \%$. See Table 1 for powder composition and degree of aggregation.

sizes got slightly smaller than microscopic sizes, indicating that the balance between particle dispersion and fragmentation leaned towards fragmentation. Nevertheless, the strongly agglomerated powder (powder D) could not be dispersed by a pressure of 3 bar, but needed up to 5 bar to reach a diameter similar to the light microscopy reference (Figure 4). A narrow unim-

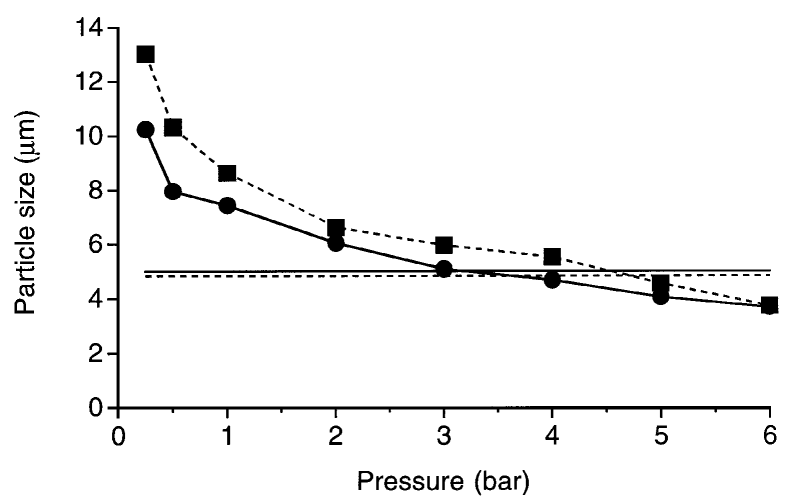

Figure 4. Comparison between laser diffraction (LD) following dry dispersion and light microscopy (LM) for the analysis of particle Size of powders B' and D'. LD measurements were performed with pressures of compressed air varying from 0.25 to 6 bar. Key: (-) powder B'; (--) powder D'. The straight lines are the reference LM mass median particle sizes. The coefficients of variation for the LD sizes collected at a pressure of 3 bar ranged between 2 and 6\%. See Table 1 for powder composition and degree or aggregation. odal mass distribution size profile following dry dispersion did not mean that there were no aggregates or particle breaking, but a shoulder or a bimodal distribution did indicate incomplete deaggregation and/or particle breaking.

Without ultrasounds, the mass median diameters obtained by laser diffraction after suspension in water were up to six times higher than the mass median diameters from microscopy (Figure 5a). A sonication period of $30 \mathrm{~s}$ at a power

\section{a}

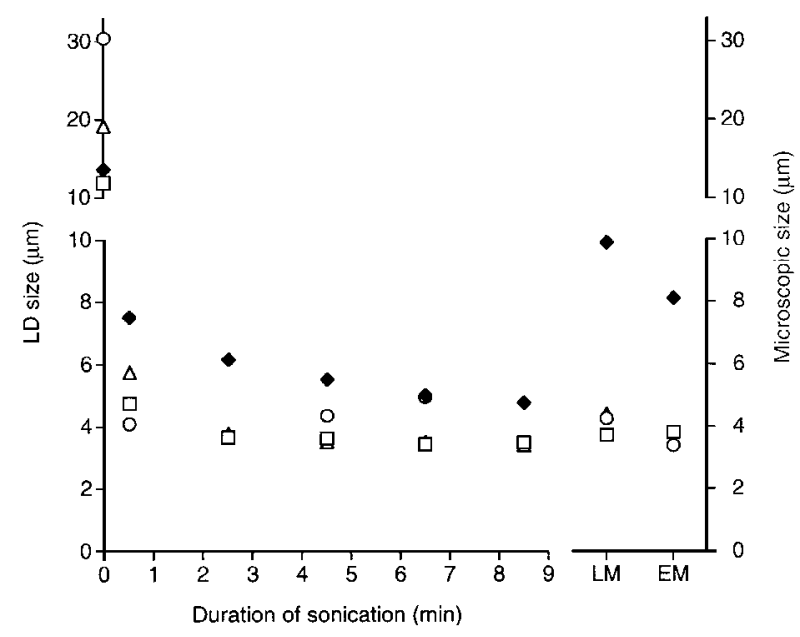

b

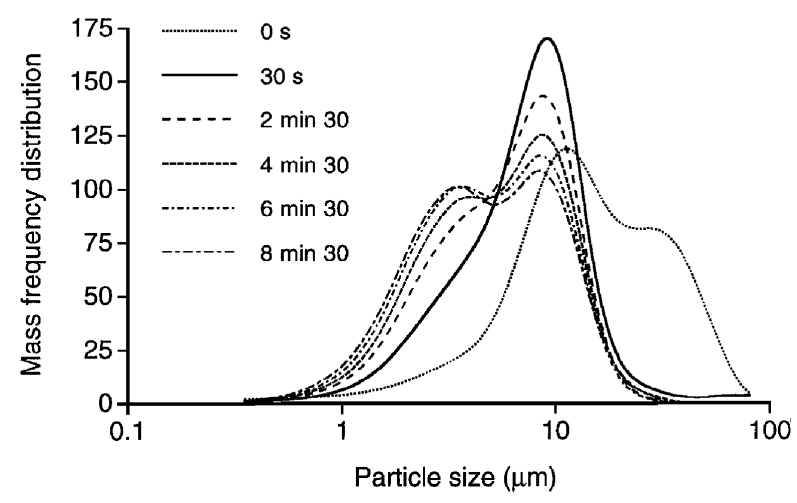

Figure 5. (a) Comparison between laser diffraction (LD) following suspension in water and ultrasonic dispersion and light (LM) and electron (EM) microscopy for the analysis of particle size of powders A, B, C, and D. Increasing periods of sonication (from $0 \mathrm{~s}$ to $8.5 \mathrm{~min}$ ) were applied before measurement. Key: $(\diamond)$ mass median particle size of powder A; $(\square)$ powder B; $(\triangle)$ powder $\mathrm{C}$; and $(\mathrm{O})$ powder $\mathrm{D}$. Coefficients of variation for measurements performed following $30 \mathrm{~s}$ of sonication were $7 \%$. (b) Mass distribution size profiles of powder A collected with laser diffraction in the wet state following increasing periods of sonication. See Table 1 for powder composition and degree of aggregation. 
of $60 \mathrm{~W}$ was sufficient to consistently deaggregate the powders, and the sizes reached those obtained by microscopy, even in the case of strongly agglomerated powders $(p>0.05$ for powders A, $\mathrm{B}$, and $\mathrm{D})$. After $30 \mathrm{~s}$ of ultrasonic dispersion, the mass distribution size profiles of the four powders also became narrow and unimodal. Further sonication slightly decreased particle size (Figure 5a). The mass distribution size profile of powder A collected following increasing periods of sonication is shown in Figure 5b. Beyond $30 \mathrm{~s}$, particles with diameters smaller than these measured by microscopy appeared in the size distribution profile and the number of large ones decreased, making the distribution bimodal. This result suggests that particle breaking and/or reorganization of DPPC as submicrometer-size vesicles (data not shown) occurred for sonication periods $>30 \mathrm{~s}$.

\section{Comparison of Particle Size Analysis Techniques Used in Recommended Conditions}

A comparison of the particle sizes of the four powders, collected with the different techniques in recommended conditions, is shown in Figure 6. In the case of powders A, B, and C (i.e., slightly to moderately aggregated powders), the mass mode

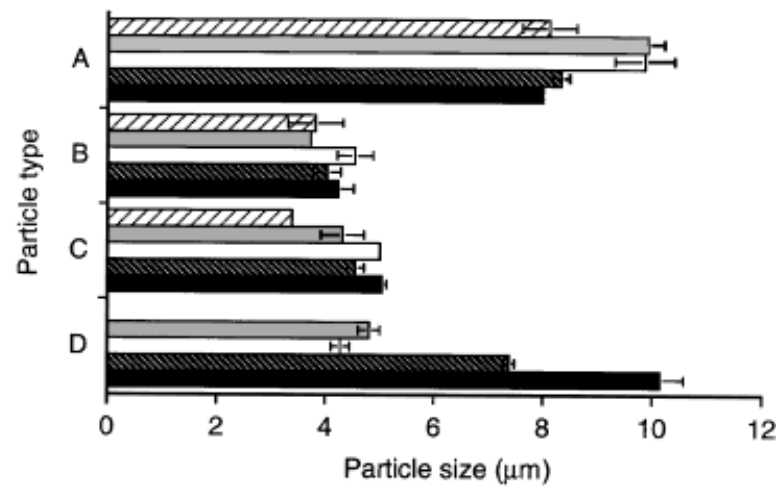

Figure 6. Comparison of particle size analysis techniques used in recommended conditions for powders $\mathrm{A}$, B, C, and D. Key: (open bar with black slashmarks) electron microscopy mean median particle diameter; (light shaded bar) light microscopy mass median particle diameter; (open bar) laser diffraction mass median particle diameter collected in the wet state following $30 \mathrm{~s}$ of ultrasonic dispersion; (dark shaded bar with white slash marks) laser diffraction mass median particle diameter collected in the dry state at a pressure of 3 bar; (dark shaded bar) electrical zone sensing mass mode particle diameter. See Table 1 for powder composition and degree of aggregation. obtained by electrical zone sensing and the mass median measured by laser diffraction at a pressure of $3 \mathrm{bar}$ or following $30 \mathrm{~s}$ of sonication were similar to the mass median evaluated by either electron or light microscopy. Electron microscopy, electrical zone sensing, and laser diffraction in the dry state failed to give an adequate estimation of the size of powder D (i.e., the strongly agglomerated powder), and laser diffraction measurement in the wet state and following $30 \mathrm{~s}$ of sonication was the only technique capable of deaggregating the powder and providing a size equal to the light microscopy reference.

\section{DISCUSSION}

The aim of this work was to compare techniques for primary particle size analysis in the case of inhalation dry powders, considering microscopy as a reference, and to determine the appropriate conditions (e.g., the conditions of dispersion) for exact measurement. Both electrical zone sensing and laser diffraction analysis following dispersion in the dry or wet state resulted in a good assessment of primary particle size of free-flowing as well as moderately aggregated dry powders. In the case of very cohesive powders, only laser diffraction after suspension in water and ultrasonic dispersion was capable of producing an exact measurement.

Microscopy was considered as the reference technique because it allows one to distinguish the individual particles from the aggregates. However, measurement by microscopy presents several drawbacks: (i) it is extremely time-consuming; (ii) the number of particles counted is small and accuracy is therefore reduced, as shown by the larger variability in measurement compared with the automated methods (see the coefficients of variation in Table 1, Figures 3-5); and (iii) although microscopy allows to visualize the particles, the particle sizes are collected from one side of the particles and not from their total volumes, which can induce inaccuracy in the calculation of the mass median diameter. In contrast to light microscopy, electron microscopy allows one to collect sharp images of particles shape and surface. However, this technique was not suitable for the size measurement of strongly cohesive powders, and coating of the samples with gold might partly alter the aspect of the particles. Light microscopy was more rapid than electron microscopy and could be used for the estimation of 
size of all the powders investigated. However, particles $<1 \mu \mathrm{m}$ could not be detected, which might create an overestimation in particle size (Table 1).

An accurate measurement of the size of slightly to moderately aggregated powders could be obtained by the mass mode of electrical zone sensing because, in contrast to the mass median, it was not affected by the aggregation state of the powder. However, the mass mode was completely aberrant in the case of strongly cohesive powders. Two important limitations affect, therefore, the analysis by electrical zone sensing: (i) no controlled means of powder deaggregation is associated with the device; and (ii) because measurements are performed in an aqueous solution, the method is only applicable to water-insoluble particles. In our case, the water-soluble excipients, albumin, lactose, and mannitol, dissolved in the medium, and the size was principally due to the lipophilic excipient, DPPC. Surprisingly, the water-soluble excipients affected the mass size distribution profiles (Figure 1), suggesting that their dissolution was not total. The mass mode obtained by electrical zone sensing was close to the mass median diameter obtained by laser diffraction in the dry state at a pressure of $3 \mathrm{bar}$ for powders A, B, and C (Figure 6;p<0.05 for powder $\mathrm{A}, p>0.05$ for powders $\mathrm{B}$ and $\mathrm{C}$ ) as well as for particles prepared with $20 \%$ DPPC (data not shown). These results suggest that, in spite of the dissolution of the hydrophilic excipients, no important decrease in particle size occurred.

The mass median particle diameter measured by laser diffraction following dispersion with compressed air at a pressure of 3 bar gave a correct estimation of the size of free-flowing as well as moderately aggregated powders (Figure 4). This pressure presented a good compromise between the separation of the agglomerates and the breaking of the particles. However, very cohesive powders could not be deaggregated by a pressure of 3 bar, and 5 bar probably involved both increased deaggregation and significant particle breaking, as suggested by the bimodal mass distribution size profile. Analysis by laser diffraction in the dry state presents the major advantage of measuring the size of the whole particles without the alterations in shape and volume and/or dissolution characteristic of the contact with a liquid medium. This advantage is particularly important in the case of powders made of a combination of hydrophilic and lipo- philic excipients as described in this article. However, the pressure necessary for powder dispersion in primary particles could not be determined from the mass distribution size profiles or from the steepness of the decrease in particle size as a function of pressure (Figure 4) but required comparison with microscopy for each powder type.

Laser diffraction analysis after suspension in water needed a sonication period of $30 \mathrm{~s}$ at a power of $60 \mathrm{~W}$ to totally disperse the powders and produce a good assessment of particle size (Figure 5a). Beyond $30 \mathrm{~s}$, particle fragmentation occurred (Figure 5b). Laser diffraction in the wet state was the only method applicable to highly and less respirable powders. This measurement is also associated with the dissolution of a part of the particles, but in contrast to electrical zone sensing, a standardized means of sample deaggregation can be used before analysis.

Our study points out the particular need to validate particle sizing methods as a function of particle material as well as powder dispersion through the use of multiple complementary techniques. We underlay the limitations of the methods in the case of inhalation dry powders and show that they mainly involve improper deaggregation. In contrast to previous studies, ${ }^{13-15}$ we also reach recommendations for proper use by comparing each technique to microscopy.

\section{ACKNOWLEDGMENTS}

The authors thank Patrick Van Der Smissen for training with the electron microscope and the Pharmacotechnology and Biopharmaceutics Laboratory (Katholieke Universiteit Leuven) for the use of the electrical zone sensing instrument. Cynthia Bosquillon was supported by a fellowship from Université catholique de Louvain (UCL). Rita Vanbever is appointed by the Fonds National de la Recherche Scientifique (FNRS, Belgium). This work was funded in part by the FNRS and UCL Special Funds for Research.

\section{REFERENCES}

1. Martonen T, Yang Y. 1996. Deposition mechanics of pharmaceutical particles in human airways. In: Hickey AJ, editor. Inhalation aerosols. New York: Marcel Dekker. pp. 3-27.

2. Hinds WC. 1999. Uniform particle motion. In: Hinds WC, editor. Aerosol technology-Properties, 
behavior, and measurement of airborne particles, $2^{\text {nd }}$ ed. New York: John Wiley \& Sons. pp. 42-74.

3. Gerrity TR, Lee PS, Hass FJ, Marinelli A, Werner P and Lourenço RV. 1979. Calculated deposition of inhaled particles in the airway generations of normal subjects. J Appl Physiol 47:867-873.

4. Hinds WC. 1999. Respiratory deposition. In: Hinds WC, editor. Aerosol technology-Properties, behavior, and measurement of airborne particles, $2^{\text {nd }}$ ed. New York: John Wiley \& Sons. pp. 233-259.

5. Préparations pour inhalation: Evaluation aérodynamique des particules fines - Dose des particules fines et distribution granulométrique des particules. In: European Pharmacopoeia, $3^{\text {rd }}$ ed. Strasbourg, Addendum 2001, pp 115-126.

6. Physical tests and determination: Aerosols. 1995. In: United States Pharmacopoeia 23, pp. 17601767.

7. Méthodes de pharmacotechnie. 1996. In: European Pharmacopoeia, $3^{\text {rd }}$ ed. Strasbourg, pp. 141-142.

8. Martin A. 1993. Micromeritics. In: Martin A, editor. Physical pharmacy: Physical chemical principles in the pharmaceutical sciences, $4^{\text {th }}$ ed. London, Philadelphia: Lea \& Febiger, pp. 423-449.

9. Washington C., editor. 1992. Particle size analysis in pharmaceutics and other industries-Theory and practice, Chichester, England: Ellis Horwood Limited.

10. Vanbever R, Mintzes JD, Wang J, Nice J, Chen D, Batycky R, Langer R, Edwards DA. 1999. Formulation and physical characterization of large porous particles for inhalation. Pharm Res 16: 1735-1742.

11. Bosquillon C, Lombry C, Préat V and Vanbever R. 2001. Influence of formulation excipients and physical characteristics of inhalation dry powders on their aerosolization properties. J Controlled Release 70:329-339.

12. Edwards DA, Hanes J, Caponetti G, Hrkach J, Ben-Jebria A, Eskew ML, Mintzes JD, Deaver D, Lotan N, Langer R. 1997. Large porous particles for pulmonary drug delivery. Science 276:18681871.

13. De Villiers MM. 1995. Influence of cohesive properties of micronized drug powders on particle size analysis. J Pharm Biomed 13:191-198.

14. Etzler FM, Sanderson MS. 1995. Particle size analysis: A comparative study of various methods. Part Syst Charact 12:217-224.

15. Iacocca RG, German RM. 1997. A comparison of powder particle size measuring instruments. Int $\mathrm{J}$ Powder Metall 33:35-48. 